\title{
Enhancement of Security through a Cryptographic Algorithm Based on Mathematical Representation of Natural Numbers
}

\author{
${ }^{1}$ Pulkit Singhal, ${ }^{2}$ Narendra Kumar Verma, ${ }^{3}$ Aurobinda Laha, ${ }^{4}$ Prof. Pranam Paul and ${ }^{5}$ Prof. A K \\ Bhattacharjee
}

\begin{abstract}
The paper deals with a new algorithm, which is based on mathematical idea. It is time efficient and easily understandable, as, it is the scheme used in cipher mathematics based. Here at encryption we consider cipher text as natural numbers and then the encrypted message is obtained by taking the cube, subtraction, division, hence for which we got a factor which is then transmitted, and then at decryption the reverse i.e. multiplication and cube root. In short we can say it as Mathematical data encryption and decryption (MDED). The decryption algorithm will be there for the receiver as the private key.
\end{abstract} key

Index Terms - Encryption, cipher text, Decryption, private

\section{INTRODUCTION}

Cryptography is the practice and study of hiding information. Modern Cryptography intersects the disciplines of mathematics, computer science and engineering. Applications of cryptography include ATM cards, computer passwords, and electronic commerce. In this paper, a mathematics-based, private-key, which we can call as decipher or decryption algorithm, is presented, which is modeled for implementing each character with a natural number.

Section 2 explains the algorithm of encryption and decryption of the text read from the file specified in the code itself. Section 3 describes an implementation with the help of a real example. Section 4 gives the Results related to the Algorithm which involves Execution Time and type of files on which it can be implemented. Section 5 comprises an analytical and conclusive discussion of the technique.

\section{THE SCHEME}

The algorithm of encryption and decryption technique of a

${ }^{1,2,3}$ Student, ECE Dept, National Institute of Technology, Durgapur 713209, India

${ }^{4}$ Dept of Computer Application, Dr. B. C. Roy Engineering College, Durgapur - 713209, India

${ }^{5}$ ECE Dept, National Institute of Technology, Durgapur - 713209, India 1pulkitsinghalraj@gmail.com, 3aurobinda.nitdgp@gmail.com,

5akbece12@yahoo.com text message used during implementation of natural number algorithm is presented as follows.

\section{A. Encryption Technique:}

Step 1:- To encrypt a text message at first the given text is stored in a string variable, say $\mathbf{m s g}$.

Step 2:- The length of the message is stored in a numeric variable $\boldsymbol{x} \boldsymbol{1}$.

Step 3:- A one-dimensional array $\boldsymbol{e}$ 1/ / represents the equivalent coded natural number for each of the alphabets with a character for space in the string variable msg.

TABLE 2.1 EquiValent NATURAL NuMBer For EACH OF CHARACTER

\begin{tabular}{|c|c|c|}
\hline $\begin{array}{c}\text { Serial } \\
\text { No. }\end{array}$ & Character & Equivalent Natural Number \\
\hline 1. & $\mathrm{a}$ & 1 \\
\hline 2. & $\mathrm{~b}$ & 2 \\
\hline 3. & $\mathrm{c}$ & 3 \\
\hline 4. & $\mathrm{~d}$ & 4 \\
\hline 5. & $\mathrm{e}$ & 5 \\
\hline 6. & $\mathrm{f}$ & 6 \\
\hline 7. & $\mathrm{~g}$ & 7 \\
\hline 8. & $\mathrm{~h}$ & 8 \\
\hline 9. & $\mathrm{i}$ & 9 \\
\hline 10. & $\mathrm{j}$ & 10 \\
\hline 11. & $\mathrm{k}$ & 11 \\
\hline 12. & 1 & 12 \\
\hline 13. & $\mathrm{~m}$ & 13 \\
\hline 14. & $\mathrm{n}$ & 14 \\
\hline 15. & o & 15 \\
\hline
\end{tabular}




\begin{tabular}{|c|c|c|}
\hline 16. & $\mathrm{p}$ & 16 \\
\hline 17. & $\mathrm{q}$ & 17 \\
\hline 18. & $\mathrm{r}$ & 18 \\
\hline 19. & $\mathrm{~s}$ & 20 \\
\hline 20. & $\mathrm{t}$ & 21 \\
\hline 21. & $\mathrm{u}$ & 22 \\
\hline 22. & $\mathrm{v}$ & 23 \\
\hline 23. & $\mathrm{w}$ & 24 \\
\hline 24. & $\mathrm{x}$ & 25 \\
\hline 25. & $\mathrm{y}$ & 26 \\
\hline 26. & $\mathrm{z}$ & 27 \\
\hline 27. & space & \\
\hline
\end{tabular}

Step 4:- Each of the numbers in the array e1[ ] are cubed and stored in a numeric array e2[].

Step 5:- Then the array e1[ ] is subtracted from e2[ ] and kept in array e3[ ].

Step 6:- Each of the numbers in the numeric array e3 [ ] is divided by 6 and the factors obtained are stored in the array cipher_text[ ]

Step 7:- The array cipher_text/] represents the encrypted message which is then sent to the receiver.

\section{B. eNcryption Technique:}

Step 1:- Initially after getting the encrypted message the receiver will use the private key i.e the decryption algorithm to get back the original message. Here we use the array cipher_text to decrypt the message received.

Step 2:- Each of the numbers in array cipher_text is multiplied by 6 and stored in the numeric array d1[ ].

Step 3:- We will take an array b1 [] which will comprise of the cubes of first 27 natural numbers corresponding to each of the alphabets including a for a space.

Step 4:- Then we will run a loop in which we subtract the respective array $d 1[$ ] with $b 1[$ ] and keep the result in array c1[]

Step 5:- Then we will take the magnitude of the array c1[ ] and keep in the array g1[ ].

Step 6:- The receiver will take the minimum of the numbers in array $\mathrm{g} 1[\mathrm{]}$.

Step 7:-This array g1[ ] is the final array from which the decrypted message is obtained.

\section{IMPLEMENTATION AND THE CODE AS DESIGNED IN MATLAB}

For example we consider a message "gandhiji was great" to be sent. Now each character is decomposed into a natural number including a space. In section 3.1, encryption is discussed, in 3.2 the key generation part is shown and finally in section 3.3 , decryption is shown.

\section{A. Encryption}

Here each character is replaced by one natural number starting from lets say 1 . So the encrypted characters as natural numbers are shown in table 3.1.1.

TABLE 3.1.1 TABLE SHOWING THE ENCRYPTION OF EACH AlPHABET

\begin{tabular}{|c|c|c|c|}
\hline Text & Serial no. & letter & $\begin{array}{c}\text { Replaced } \\
\text { number }\end{array}$ \\
\hline \multirow{18}{*}{$\begin{array}{c}\text { gandhiji } \\
\text { was } \\
\text { great }\end{array}$} & 1 & g & 7 \\
\hline & 2 & $\mathrm{a}$ & 1 \\
\hline & 3 & $\mathrm{n}$ & 14 \\
\hline & 4 & $\mathrm{~d}$ & 4 \\
\hline & 5 & $\mathrm{~h}$ & 8 \\
\hline & 6 & $\mathrm{i}$ & 9 \\
\hline & 7 & $\mathrm{~J}$ & 10 \\
\hline & 8 & $\mathrm{i}$ & 9 \\
\hline & 9 & space & 27 \\
\hline & 10 & $\mathrm{~W}$ & 23 \\
\hline & 11 & $\mathrm{a}$ & 1 \\
\hline & 12 & $\mathrm{~s}$ & 19 \\
\hline & 13 & space & 27 \\
\hline & 14 & $\mathrm{~g}$ & 7 \\
\hline & 15 & $\mathrm{r}$ & 18 \\
\hline & 16 & $\mathrm{e}$ & 5 \\
\hline & 17 & $\mathrm{a}$ & 1 \\
\hline & 18 & $\mathrm{t}$ & 20 \\
\hline
\end{tabular}

So after replacing each character and space through a natural number we use the property of natural number that any number when cubed and subtracted by the number itself and hence divided by 6 gives the remainder

\section{B. Key Generation}

So now when we do the process of cubing, subtracting and hence the division the final factor on division left is our key which is sent to the receiver. The generated key is shown in table 3.2.1

TABLE 3.2.1 TABLE SHOWING THE KEY SENT

\begin{tabular}{|c|c|c|}
\hline Serial no. & Character & Sent key \\
\hline 1 & $\mathrm{~g}$ & 56 \\
\hline 2 & $\mathrm{a}$ & 0 \\
\hline 3 & $\mathrm{n}$ & 455 \\
\hline 4 & $\mathrm{~d}$ & 10 \\
\hline 5 & $\mathrm{~h}$ & 84 \\
\hline 6 & $\mathrm{i}$ & 120 \\
\hline 7 & $\mathrm{j}$ & 165 \\
\hline 8 & $\mathrm{i}$ & 120 \\
\hline 9 & $\mathrm{space}$ & 3276 \\
\hline 10 & $\mathrm{w}$ & 2024 \\
\hline 11 & $\mathrm{a}$ & 0 \\
\hline 12 & $\mathrm{~s}$ & 1140 \\
\hline 13 & $\mathrm{space}$ & 3276 \\
\hline 14 & $\mathrm{~g}$ & 56 \\
\hline 15 & $\mathrm{r}$ & 969 \\
\hline 16 & $\mathrm{e}$ & 120 \\
\hline 17 & $\mathrm{a}$ & 1330 \\
\hline 18 & $\mathrm{t}$ & \\
\hline & & 0 \\
\hline
\end{tabular}

\section{Decryption}

After we have got the key we do the reverse process i.e we multiply the key with 6 and then we see which number is coming as a perfect cube of any natural number around the number got by multiplying by 6 .

Mathematically we multiply the key with 6 and then subtract the number from a set of cubes of 27 numbers. Then 
we take the magnitude of the resultant and hence the minimum of all the numbers will be our final number to be obtained. A detail is shown in table 3.3.1

TABLE 3.3.1 TABLE Showing THE DeCRyption TABLE IN DetAIL

\begin{tabular}{|r|r|r|r|c|}
\hline $\begin{array}{c}\text { Sl } \\
\text { no. }\end{array}$ & $\begin{array}{c}\text { Key } \\
* \\
\mathbf{6}\end{array}$ & Matched cube & Final number & $\begin{array}{c}\text { Encrypted } \\
\text { Character }\end{array}$ \\
\hline 1 & 336 & 343 & 7 & $\mathrm{~g}$ \\
\hline 2 & 0 & 1 & 1 & $\mathrm{a}$ \\
\hline 3 & 2730 & 2744 & 14 & $\mathrm{n}$ \\
\hline 4 & 60 & 64 & 4 & $\mathrm{~d}$ \\
\hline 5 & 504 & 512 & 8 & $\mathrm{~h}$ \\
\hline 6 & 720 & 729 & 9 & $\mathrm{i}$ \\
\hline 7 & 990 & 1000 & 10 & $\mathrm{j}$ \\
\hline 8 & 720 & 729 & 9 & $\mathrm{i}$ \\
\hline 9 & 19656 & 19683 & 27 & space \\
\hline 10 & 12144 & 12167 & 23 & $\mathrm{w}$ \\
\hline 11 & 0 & 1 & 1 & $\mathrm{a}$ \\
\hline 12 & 6840 & 6859 & 19 & $\mathrm{~s}$ \\
\hline 13 & 19656 & 19683 & 27 & space \\
\hline 14 & 336 & 343 & 7 & $\mathrm{~g}$ \\
\hline 15 & 5814 & 5832 & 18 & $\mathrm{r}$ \\
\hline 16 & 720 & 125 & 5 & $\mathrm{e}$ \\
\hline 17 & 0 & 1 & 1 & $\mathrm{a}$ \\
\hline 18 & 7980 & 8000 & 20 & $\mathrm{t}$ \\
\hline
\end{tabular}

Decrypted massage is "gandhiji was great" which is same as original massage.

\section{Results}

Here we have ciphered each letter into some natural numbers and hence got keys and hence decrypted the keys to obtain the final character and hence the final message. We have also shown the execution time i.e time taken to execute the number of characters as per the length. We have seen that this algorithm works on all type of text files of any sizes as per the number of characters the file contains. It is shown in Table 4.1.

TABLE 4.1 COMPARISON OF THE EXECUTION TIME FOR TEXTS VARYING IN LENGTH

\begin{tabular}{|r|r|}
\hline \multicolumn{1}{|c|}{$\begin{array}{l}\text { Number of letters in the text including } \\
\text { blank spaces and special characters }\end{array}$} & \multicolumn{1}{c|}{$\begin{array}{c}\text { Execution time } \\
\text { in seconds }\end{array}$} \\
\hline 0 & 0 \\
\hline 1 & 0.019235 \\
\hline 8 & 0.020015 \\
\hline 16 & 0.021678 \\
\hline 32 & 0.022875 \\
\hline 40 & 0.024565 \\
\hline 48 & 0.028963 \\
\hline 56 & 0.033455 \\
\hline 64 & 0.038676 \\
\hline 72 & 0.042459 \\
\hline 80 & 0.046521 \\
\hline 88 & 0.050983 \\
\hline 96 & 0.055433 \\
\hline 100 & 0.069873 \\
\hline
\end{tabular}

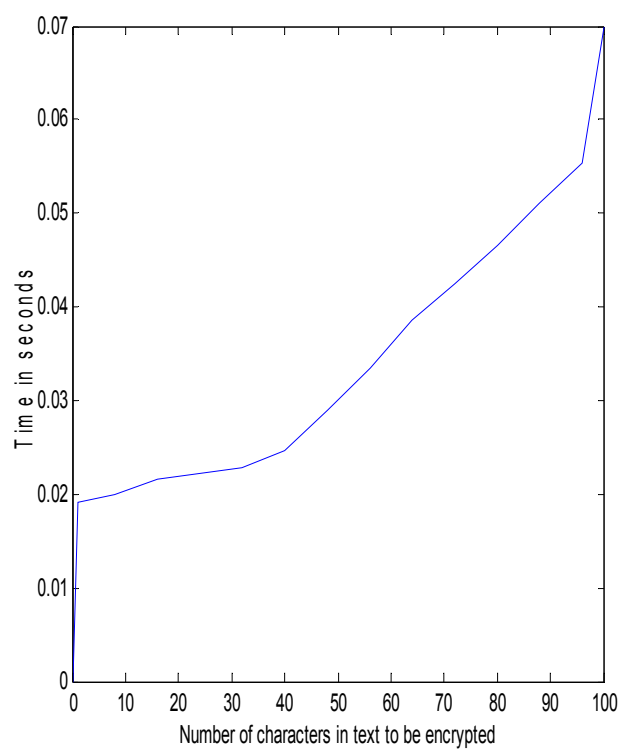

Figure 4.1 A Relationship between execution time and Text length

\section{ANALytical Discussion AND CONCLUSION OF THE USED ALGORITHM}

Using this Decryption NNA (Natural number algorithm) private key technique, we can encrypt any size of file, as well as any kind of file, as NNA protocol can be implemented to any file as each of the characters are represented by natural numbers so any type of message can be sent. There might be little bit more time taken to execute but still it is a very efficient algorithm as it is purely mathematics based and mathematics leads us to everywhere and moreover memory required is less. so having this easy and far more efficient algorithm with a great security aspect gives it a new shape and dimension in this era. The enhanced security is due to the peculiarity of the different manipulations and properties used in the algorithm which is quite incomprehensible to unauthorized person.

The only disadvantage is that it doesn't work on video files.

Time taken by the algorithm will depend on the platform on which the user is working.

\section{REFERENCES}

[1] J. K. Mandal, S. Dutta, "A 256-bit recursive pair parity encoder for encryption", Advances D -2004, Vol. $9 \mathrm{n}^{\circ} 1$, Association for the Advancement of Modelling and Simulation Techniques in Enterprises (AMSE, France),

[2] www. AMSE-Modeling.org, pp. 1-14.

[3] Pranam Paul, Saurabh Dutta, "A Private-Key Storage-Efficient Ciphering Protocol for Information Communication Technology", National Seminar on Research Issues in Technical Education (RITE), March 08-09, 2006, National Institute of Technical Teachers' Training and Research, Kolkata, India.

[4] Pranam Paul, Saurabh Dutta, "An Enhancement of Information Security Using Substitution of Bits Through Prime Detection in Blocks", Proceedings of National Conference on Recent Trends in Information Systems (ReTIS-06), July 14-15, 2006, Organized by IEEE Gold Affinity Group, IEEE Calcutta Section, Computer Science \& Engineering Department, CMATER \& SRUVM Project- Jadavpur University and Computer Jagat. 
[5] Dutta S. and Mandal J. K., "A Space-Efficient Universal Encoder for Secured Transmission", International Conference on Modelling and Simulation (MS' 2000 -Egypt, Cairo, April 11-14, 2000.

[6] Mandal J. K., Mal S., Dutta S., A 256 Bit Recursive Pair Parity Encoder for Encryption, accepted for publication in AMSE Journal, France, 2003.

[7] Dutta S., Mal S., "A Multiplexing Triangular Encryption Technique A move towards enhancing security in ECommerce", Proceedings of IT Conference (organized by Computer Association of Nepal), 26 and 27 January, 2002, BICC, Kathmandu IJCSNS International Journal of Computer Science and Network Security, VOL.8 No.4, April 2008.

[8] William Stallings, Cryptography and Network security: Principles and practice (Second Edition), Pearson Education Asia, Sixth Indian Reprint 2002.

[9] Atul Kahate (Manager, i-flex solution limited, Pune, India), Cryptography and Network security, Tata McGraw-Hill Publishing Company Limited.

[10] Mark Nelson, Jean-Loup Gailly, The Data Compression Book. BPB Publication.

[11] S Mal, J K Mandal and S Dutta, "A Microprocessor Based Encoder for Secured Transmission", Conference on Intelligent Computing on VLSI, Kalyani Govt. Engineering College, 1-17 Feb, 2001, pp 164-169.

[12] Pranam Paul, Saurabh Dutta, A. K. Bhattacherjee, "An Approach to ensure Security through Bit-level Encryption with Possible Lossless Compression", International Journal of Computer Science and Network Security, Vol. 8 No. 2, pp 291-299.

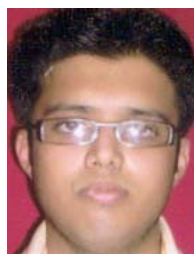

Pulkit Singhal is a student pursuing his B.Tech degree (final year) in Electronics and Communication Engineering from National Institute of Technology (NIT), Durgapur, West Bengal,India.His current areas of research is Networking and VLSI design.

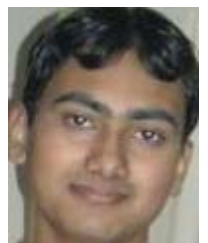

Narendra Kumar Verma is a student pursuing his B.Tech degree (final year) in Electronics and Communication Engineering from National Institute of Technology (NIT), Durgapur, West Bengal, India.His current areas of research is Speech processing and cryptography.

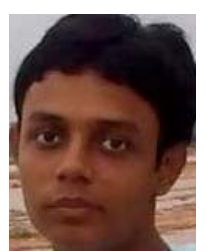

Aurobinda Laha is a student pursuing his B.Tech degree (final year) in Electronics and Communication Engineering from National Institute of Technology (NIT), Durgapur, West Bengal, India.His current areas of research is Information Securty,VLSI and Microwave circuits.

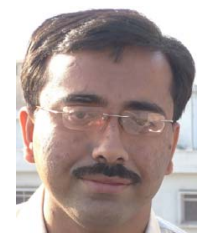

Prof. Pranam Paul is associated withDepartment of MCA of Dr. B. C. Roy Engineering College, Durgapur, West Bengal, INDIA in the. He has already submitted his $\mathrm{Ph}$.D. thesis in ECE department NIT, Durgapur in the field of Cryptography and Network Security. He has total 19 research publications

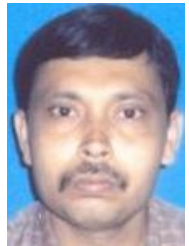

A.K. Bhattacharjee did his Ph.D. in Engineering from Jadavpur University, Kolkata, India in 1989. Presently he is associated with Department of ECE in NIT, Durgapur, INDIA. The senior academicians, having been involved in teaching and research for last 20 years, his current areas of research are Microstrip Antenna and cryptography 